Simultaneous distributed strain and temperature sensing based on combined Raman-Brillouin scattering using Fabry-Perot lasers

This article has been downloaded from IOPscience. Please scroll down to see the full text article.

2010 Meas. Sci. Technol. 21094025

(http://iopscience.iop.org/0957-0233/21/9/094025)

View the table of contents for this issue, or go to the journal homepage for more

Download details:

IP Address: 128.178.23.47

The article was downloaded on 01/11/2012 at 15:21

Please note that terms and conditions apply. 


\title{
Simultaneous distributed strain and temperature sensing based on combined Raman-Brillouin scattering using Fabry-Perot lasers
}

\author{
Gabriele Bolognini, Marcelo A Soto and Fabrizio Di Pasquale \\ Scuola Superiore Sant'Anna, via G Moruzzi 1, 56124 Pisa, Italy \\ E-mail: gabriele.bolognini@sssup.it
}

Received 14 January 2010, in final form 4 May 2010

Published 28 July 2010

Online at stacks.iop.org/MST/21/094025

\begin{abstract}
An investigation is performed of the possibility of achieving simultaneous distributed strain and temperature sensing based on hybrid Raman-Brillouin scattering with the use of multi-wavelength optical sources such as common Fabry-Perot (FP) lasers. By employing a self-heterodyne detection scheme based on a multi-wavelength optical local oscillator, the benefits of FP lasers are fully exploited, allowing for high-power Raman intensity measurements and a simultaneous high-accuracy detection of the Brillouin frequency shift parameter for each FP longitudinal mode. Experimental results point out a significant reduction of coherent Rayleigh noise, and highlight the enhanced performance in hybrid Raman-Brillouin sensing when using FP lasers; in particular using standard FP lasers at $1550 \mathrm{~nm}$ results in about $12 \mathrm{~dB}(7 \mathrm{~dB})$ temperature (strain) accuracy improvement at $25 \mathrm{~km}$ sensing distance with respect to the use of standard distributed feedback lasers.
\end{abstract}

Keywords: fiber optics, sensors, Brillouin scattering, Raman scattering, fiber testing (Some figures in this article are in colour only in the electronic version)

\section{Introduction}

The fields of applications for optical fiber distributed sensing are manifold, ranging from civil and geo-technical engineering to the oil \& gas industry, and from energy management to railway and highway structures monitoring. In each of the aforementioned fields, fiber optic distributed sensors enable a variety of usages, such as structural-health monitoring (SHM) of buildings, dams and bridges, early landslide warning, leakage and strain detection in power cables and pipelines, borehole and underground mine monitoring as well as fire detection.

Distributed sensing commonly requires simultaneous measurements of temperature and deformations along the optical fiber, currently achieved by using measurement techniques based either on optical Brillouin scattering [1] or on hybrid Raman-Brillouin scattering detection [2]. Such nonlinear effects are generally exploited in measurement systems based on the optical time domain reflectometry (OTDR) principle.

In systems based on spontaneous Brillouin scattering (SpBS), strain and temperature changes can be distinguished within the same optical fiber, through a simultaneous detection of both spontaneous Brillouin intensity and Brillouin frequency shift (BFS), depending on both temperature and strain, in a scheme which requires access to one fiber-end only. Also the stimulated Brillouin scattering (SBS) effect can be exploited to measure the BFS, providing better resolution, but requiring access to both fiber ends in common implementation schemes employing Brillouin optical time-domain analysis [3] in order to measure the gain BFS. In SBS-based sensors, the issue of strain-temperature cross-sensitivity in the BFS can be overcome by using two sensing fibers, with only one fiber embedded in the structure to be monitored (thus simultaneously detecting strain and temperature variations), and the other one used to measure temperature only. Sensing 
schemes exploiting SpBS are then characterized by two main advantages with respect to systems based on SBS: the need to access one fiber-end only and the possibility of performing a simultaneous strain-temperature estimation using a single fiber. On the other hand, their performance is seriously affected by the low backscattered power values.

In hybrid schemes based on spontaneous RamanBrillouin scattering [2], conversely, the temperature profile along the sensing fiber is directly obtained by measuring the strain-independent spontaneous Raman anti-Stokes trace. The measured temperature profile is then used to obtain the fiber strain from the BFS trace, which is both temperature and strain dependent.

The main issues affecting hybrid sensors' performance are mainly related to the different Raman-Brillouin scattering cross-sections, leading to significantly different backscattered power levels and resulting in a Raman-scattering trace which is significantly lower (about one order of magnitude) and noisier with respect to the Brillouin trace. The high noise in temperature trace from spontaneous Raman scattering (SpRS) has actually been identified in the literature [2] as the main limiting factor in temperature-strain measurements when using hybrid sensing schemes.

A straightforward method to improve performance would be given by the use of higher input optical power levels. However, this is limited to low power values by the onset of nonlinear optical effects [4], such as stimulated Raman-Brillouin scattering, which distort the acquired traces. Furthermore, the common optical sources used for SpBS measurements are characterized by rather low output power levels (a few $\mathrm{dBm}$ at most); actually, in order to detect small BFS variations in the narrow-band Brillouin scattering (which is few tens of $\mathrm{MHz}$ in silica fibers), narrowband optical sources must be used. The most commonly used sources are distributed feedback (DFB) lasers or external cavity lasers [2], which are however characterized by low output power levels (below a few tens of $\mathrm{mW}$ ).

Hence, the limitations imposed by the physics of SpBS to the maximum usable power and spectral width of the laser sources must be traded off with the need for high-power and broadband sources which can be extremely effective for the measurement of weak Raman-scattering signals.

A smart usage of commercially available optical sources, to overcome the limitations imposed by both narrowband Brillouin gain and low-level Raman-scattering light, could be of great benefit when implementing highly accurate distributed strain and temperature sensors.

In this work the use of standard Fabry-Perot (FP) lasers is proposed for achieving simultaneous distributed strain and temperature sensing based on hybrid Raman-Brillouin measurements. Such optical sources, which are inherently multi-longitudinal mode sources, can be exploited to obtain enhanced hybrid sensing schemes. In order to improve the sensing performance, a novel detection scheme based on a multi-wavelength optical local oscillator (OLO) and a single photo-detector is proposed for the simultaneous measurement of the BFS for all FP modes. It is shown how the use of FP lasers provides significant accuracy improvement in both temperature and strain measurements, also overcoming the issues related to coherent Rayleigh noise, which become significant whenever using narrowband sources.

This paper is organized as follows: in section 2 a theoretical overview related to distributed hybrid RamanBrillouin sensing is presented, and a specific analysis concerning the proposed use of FP lasers for hybrid sensing is carried out. Section 3 describes in detail the used experimental set-up, while section 4 reports the attained results, also highlighting the main differences in trace analysis with respect to standard hybrid sensing employing standard single-wavelength DFB lasers. Finally, section 5 draws the conclusions and summarizes the main achievements of the proposed technique.

\section{Theory}

In hybrid Raman-Brillouin sensors for strain and temperature measurements, both SpRS and SpBS light is simultaneously detected. The temperature dependence of the anti-Stokes SpRS signal allows for a strain-independent measurement; actually, the temperature profile along the sensing fiber is directly obtained by using the ratio of the anti-Stokes Raman power $\left(P_{A S}\right)$ to the Rayleigh-backscattering power $\left(P_{B S}\right)$, which is dependent on the absolute temperature at a given fiber point according to

$$
\frac{P_{A S}}{P_{B S}} \propto\left[\exp \left(\frac{h \Delta v_{R}}{k T}\right)-1\right]^{-1},
$$

where $h$ is the Planck constant, $k$ is the Boltzmann constant, $T$ is the absolute temperature and $\Delta v_{R}$ is the separation between anti-Stokes Raman and pump light frequencies [5]. This equation should be integrated along the fiber length and Raman spectrum to take into account the wavelength-dependent loss along the light path and the cross-section spectrum of Raman anti-Stokes scattering. Assuming uniform longitudinal optical fiber characteristics and a uniform wavelength response of the optical receivers and filters, the ratio of the anti-Stokes Raman power to the Rayleigh-backscattering power as a function of the fiber temperature $T(z)$ can be written in a simplified expression as

$\frac{P_{B S}(z)}{P_{A S}(z)}=C\left\{\exp \left[\frac{h \Delta v_{R}}{k T(z)}\right]-1\right\}^{-1} \exp \{-\Delta \alpha \cdot z\}$,

where $C$ is a constant factor (incorporating the dependence on Rayleigh-backscattering coefficient and Raman gain efficiency effects) and $\Delta \alpha$ is the difference between the fiber loss values at anti-Stokes and pump wavelengths [5]. From equation (2) the fiber temperature $T(z)$ can be found once a reference temperature is known (e.g. $T(0)$ at fiber input).

In SpBS detection, fiber strain and temperature variations are inferred from measurements of BFS parameters. Changes in the distributed strain $(\Delta \varepsilon)$ at each point $z$ along the fiber can then be estimated from the BFS measurement $\left(\Delta v_{B}\right)$ related to that point, which linearly depends on both strain and temperature according to

$$
\Delta v_{B}(z)=C_{\nu B \varepsilon} \cdot \Delta \varepsilon(z)+C_{\nu B T} \cdot \Delta T(z),
$$




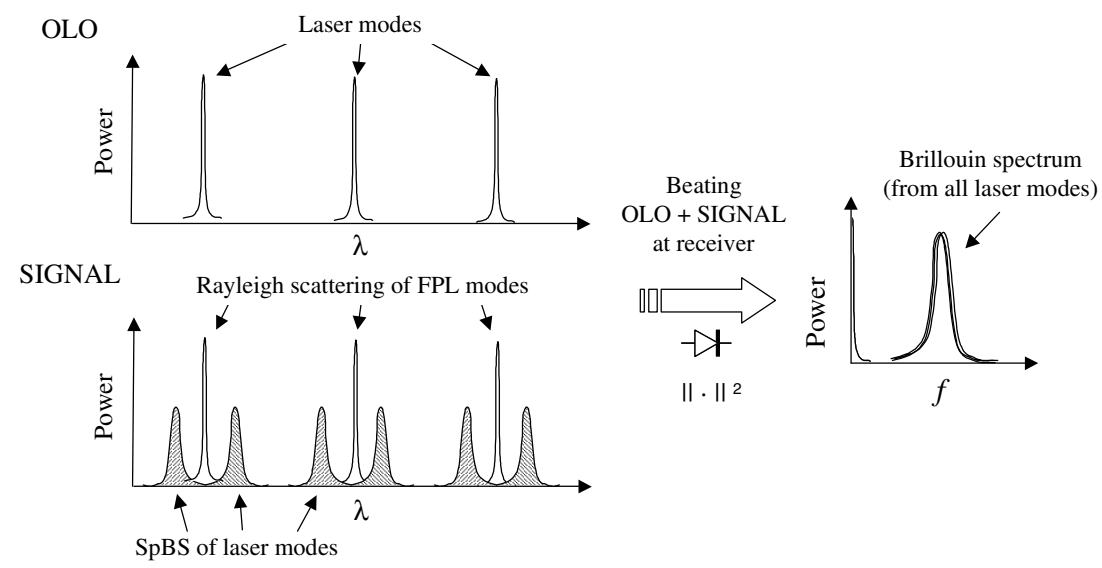

Figure 1. Principle of the scheme enabling BFS detection from individual longitudinal FP modes.

where $C_{\mathrm{\nu B} \varepsilon}=0.048 \mathrm{MHz} \mu \varepsilon^{-1}$ and $C_{\nu \mathrm{BT}}=1.07 \mathrm{MHz}^{\circ} \mathrm{C}^{-1}$ are the strain and temperature coefficients for BFS [1]. The temperature variation $\Delta T(z)$ in equation (3) is provided by equation (2), which is based on anti-Stokes Raman measurement, allowing for a temperature-independent strain estimation.

FP semiconductor lasers are inherent multi-wavelength optical sources, with a grid of equally spaced, narrowband longitudinal modes exhibiting a frequency difference depending on the laser-cavity length, typically of the order of tens of $\mathrm{GHz}$ (for common InGaAsP lasers operating at around $1550 \mathrm{~nm}$, with typical laser cavity lengths of hundreds of micrometers). FP lasers can be successfully exploited in Raman sensing, thanks to their high power (order of a few hundreds of $\mathrm{mW}$ ) and broad bandwidth, thus enabling accurate Raman measurements.

However, to our knowledge, FP lasers have not been exploited in Brillouin sensing to date, since their envelope FWHM is too broad compared to SpBS spectral width, making it impossible to accurately reconstruct the BFS parameter when using a typical heterodyne detection scheme.

Our idea is to employ FP lasers for hybrid RamanBrillouin sensing, by estimating the BFS parameter from each individual longitudinal laser mode. Actually, when a FP laser light is coupled into an optical fiber, each longitudinal mode generates SpBS. The FWHM of each FP longitudinal mode is narrower than the Brillouin gain bandwidth, allowing for an accurate BFS estimation. Also, the spectral separation among adjacent modes is typically larger than twice the BFS in silica. This allows one to clearly identify the Stokes and anti-Stokes Brillouin components for each longitudinal mode of the FP laser separately, without any interference among the adjacent modes. These features permit us to exploit the SpBS generated by all FP modes in order to obtain the BFS parameter.

Moreover, FP lasers used for SpBS measurement also possess the advantage of having a large envelope full width half maximum (FWHM, typically several $\mathrm{nm}$ ), allowing for Rayleigh-scattering measurements (required in SpBS as well as in SpRS sensing) which are not affected by coherentRayleigh noise (CRN) [6].

Note that hybrid Raman-Brillouin sensing based on single longitudinal mode lasers, e.g. DFB lasers, has been shown in the literature [2] to be limited by the Ramantemperature inaccuracy due to the low-power level of the used lasers. By exploiting the high-power capabilities of FP lasers this limitation can be overcome, providing a notably better performance in hybrid sensing.

As an additional point, while the anti-Stokes Raman intensity is measured by a simple direct-detection scheme, efficient detection in BFS measurements requires a coherentdetection receiver [2].

In parallel with the Raman direct-detection scheme, a coherent-detection scheme is proposed based on multiwavelength OLO for SpBS, enabling the simultaneous detection of BFS for all FP laser modes. The principle of such a scheme is pointed out in figure 1 . The signal arriving at receiver is essentially given by the fiber backscattering of FP light, consisting most notably in the Rayleigh-backscattering and SpBS components for each FP longitudinal laser mode. (Raman scattering is previously filtered out and used in the Raman detection scheme.) The OLO is simply given by a portion of the same FP laser light which can be tapped out before being coupled into the sensing fiber.

The beating between signal and OLO at the photodiode generates many electrical components. Those lying at lower $\mathrm{RF}$ frequencies are given by the Rayleigh-OLO beating for each mode, and the Brillouin-OLO beating for each mode. If the linewidth of OLO is small enough, the photo-detected Brillouin-OLO beating consists in a replica of the SpBS spectrum. Since those beatings occur for each FP mode, what is observed around $11 \mathrm{GHz}$, in the electric spectrum after photo-detection, is the superimposition of all SpBS spectra for each mode; this feature sensibly increases the received signal level, also enhancing the SNR of the BFS trace.

In coherent-detection schemes, the SpBS signal is amplified when mixed with an OLO, providing then a higher dynamic range compared to direct detection and the conversion of the optical Brillouin components into electric domain, leading to efficient electric spectral filtering. Finally, it has been recently demonstrated that optical pulse coding techniques provide enhanced SNR in both SpBS-based [7] and SpRS-based [5] sensors, allowing for significantly improved sensing performance. 


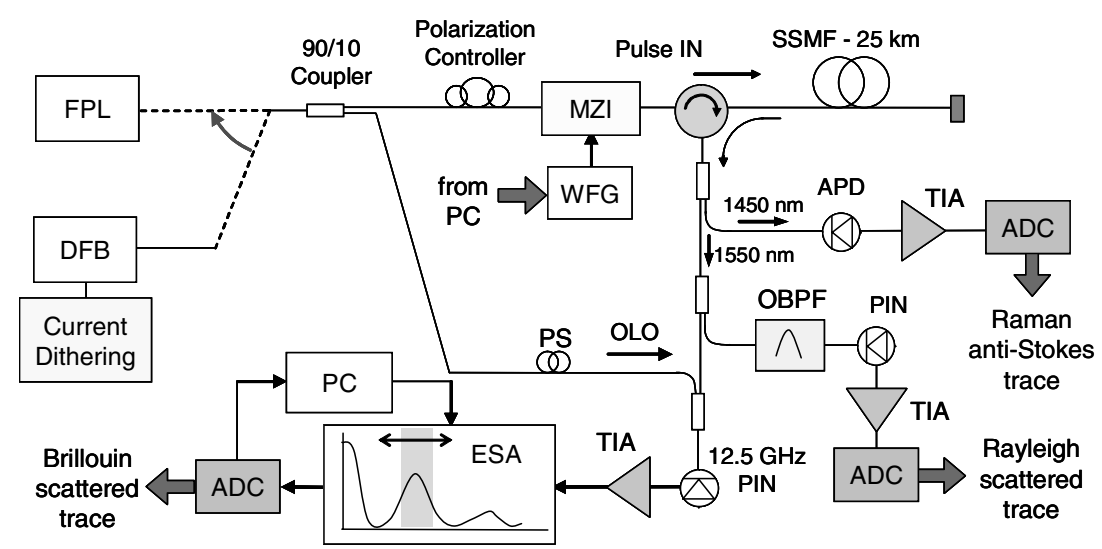

Figure 2. Experimental set-up of hybrid Raman-Brillouin distributed strain and temperature sensor.

However, the use of coding is limited in SpBS-based sensors by the SBS threshold, which constrains the maximum optical power at the fiber input [7]. In the proposed scheme employing FP lasers this limitation can be effectively overcome; the total laser power is in fact distributed among several longitudinal modes, each one being limited by the SBS threshold, thus allowing for higher total peak power levels. Due to the reduced peak power of every FP mode, the proposed technique can also be used in conjunction with optical pulse coding as well as with optical amplification (to further increase laser power) in order to further enhance the sensing performance.

\section{Experiment}

Figure 2 reports the set-up used, in which DFB and FP lasers are alternatively used in order to compare, with typical power levels, their performance for hybrid Raman-Brillouin sensing. The maximum output power of the used DFB laser is $P_{\mathrm{DFB}}=8.5 \mathrm{dBm}$ at $1550 \mathrm{~nm}$, allowing us to attain $P_{\mathrm{IN}}=$ $1.5 \mathrm{dBm}$ at the fiber input. Current dithering is also required for the DFB laser (but not for the FP laser) when measuring the Rayleigh backscattered light (which is necessary to obtain lossindependent temperature measurements) in order to reduce CRN. On the other hand, FP lasers are typically characterized by higher power levels (usually ranging from 20 to $26 \mathrm{dBm}$ ); in our experiments the power was $P_{\mathrm{FPL}}=22 \mathrm{dBm}$ at the FP laser output, allowing for $P_{\mathrm{IN}}=15 \mathrm{dBm}$ at the fiber input. As a further benefit, the total linewidth of the FP laser is large enough, thanks to the multiple longitudinal modes, so that CRN in Rayleigh measurements is inherently reduced due to wavelength averaging. The $\mathrm{CW}$-light of the source is split into two parts using a 10/90 optical coupler, so that $10 \%$ of the light is used at the receiver side as OLO and the other $90 \%$ is modulated (at the transmitter side) with $350 \mathrm{~ns}$ single pulses allowing for $\sim 35 \mathrm{~m}$ spatial resolution. Optical pulses are launched into $25 \mathrm{~km}$ of standard single mode fiber (SSMF), and the backscattered light is then coupled into the receiver side, which is composed of three stages. The first stage (Raman receiver) consists in a WDM filter separating the light band at around $1450 \mathrm{~nm}$ from the band at around $1550 \mathrm{~nm}$, so that the anti-Stokes SpRS component can be separated and coupled into an avalanche photodiode (APD) followed by a trans-impedance amplifier (TIA) and an analogdigital converter (ADC) connected to a personal computer (PC). The second stage (Rayleigh receiver), consisting in a direct-detection receiver, is used for Rayleigh-scattering measurements and detects a small portion of backscattered light at around $1550 \mathrm{~nm}$; the receiver itself is simply composed of a PIN photodiode, a TIA and an ADC connected to the PC. The third stage (Brillouin receiver) consists in an optical and electrical heterodyne receiver, including an electrical spectrum analyzer (ESA), operating in zero-span mode.

Polarization scrambling (PS) schemes have been used, for both broadband FP laser and narrowband DFB laser experiments, in order to reduce polarization-induced fading noise. When using the FP laser, a simple low-loss PS scheme [8] suitable for nm-wide optical sources has been used, exploiting a high-birefringence polarization-maintaining fiber (PMF). In such a scheme, a PMF spool, as shown in figure $3(a)$, is placed before the OLO coupling branch at the receiver, with its principal axes placed at $\pi / 4$ angle with respect to the polarized laser light, thus equally splitting the coupled laser power into the fast and slow axes, and finally resulting in a measured reduction of the degree of polarization down to $\sim 1 \%$ for the FP laser. Concerning the DFB laser, due to its significantly narrower bandwidth, a different PS scheme (characterized by higher loss than the above-mentioned PS), is required in order to significantly reduce laser polarization [9]. The implemented PS for the DFB laser, shown in figure $3(b)$, consists essentially in an unbalanced fiber Mach-Zehnder interferometer achieving phase decorrelation between the two interferometer arms, thanks to the standard single-mode fiber ( $2 \mathrm{~km}$ length) placed along one interferometer arm, and a subsequent coupling of both arms through a polarization beam combiner (PBC). With this scheme, the measured degree of polarization of CW narrow-bandwidth DFB laser was reduced below $9 \%$, so that no polarization-induced fading noise could be observed in the acquired Brillouin traces.

Note that since the lightwave spectrum of a FP laser consists of a comb of equally frequency-spaced longitudinal modes, each mode (after propagation along the fiber) generates a couple of Stokes and anti-Stokes SpBS lines, as shown in the measured spectra reported in figure 4. In order 


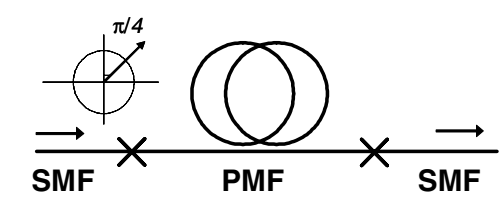

(a)

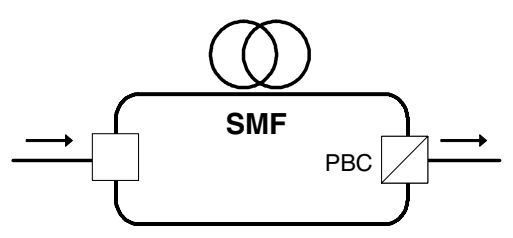

(b)

Figure 3. Outline of the used PS schemes for reducing the degree of polarization of $(a)$ FP laser and $(b)$ DFB laser.

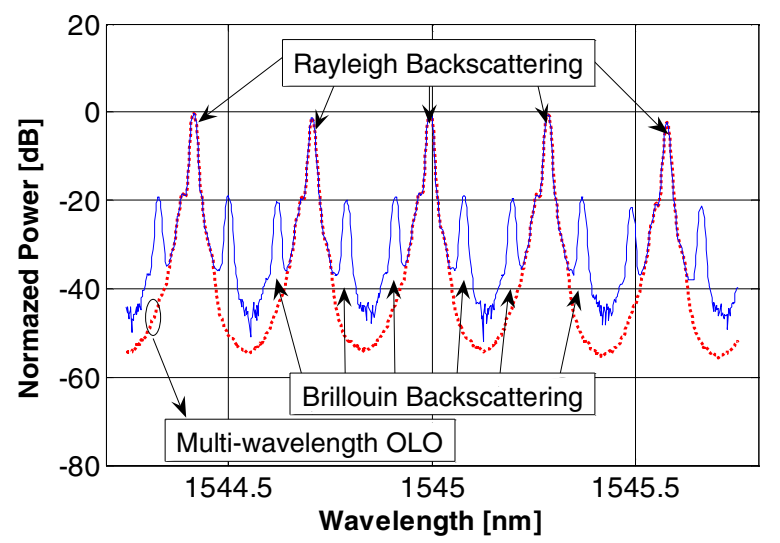

Figure 4. Spectrum of the used FP laser and respective Rayleigh, Brillouin Stokes and anti-Stokes components.

to simultaneously measure the BFS parameter for all FP longitudinal modes with a single photo-detector, the use of a multi-wavelength OLO is required. A very convenient way to provide this is to use part of the same laser light which is input into the fiber, thus allowing us also to strongly reduce issues related to phase mismatches between the source and OLO. The set-up described in figure 2 actually shows the proposed selfheterodyne detection scheme, where Brillouin components are mixed with the multi-wavelength OLO, obtained by splitting a portion of the same FP laser, before a $12.5 \mathrm{GHz}$ PIN. Figure 4 reports a zoom of the spectrum (with normalized peak power) of five modes of the used FP laser and the respective Rayleigh, Stokes and anti-Stokes SpBS components. The spectral separation between two consecutive FP longitudinal modes is $0.3 \mathrm{~nm}$, corresponding to more than twice the BFS and allowing for BFS separation among different modes. This condition actually needs to be satisfied in order to avoid possible overlapping of Stokes and anti-Stokes components of two neighboring modes.

Also, the peak mode power of the OLO needs to be much higher than the peak power of every Rayleigh backscattered mode in order to avoid distortions in the heterodyning process due to beating of Rayleigh-scattering and SpBS signals. Thus, when heterodyning the multi-wavelength OLO with the backscattered light from the fiber, only a single beating

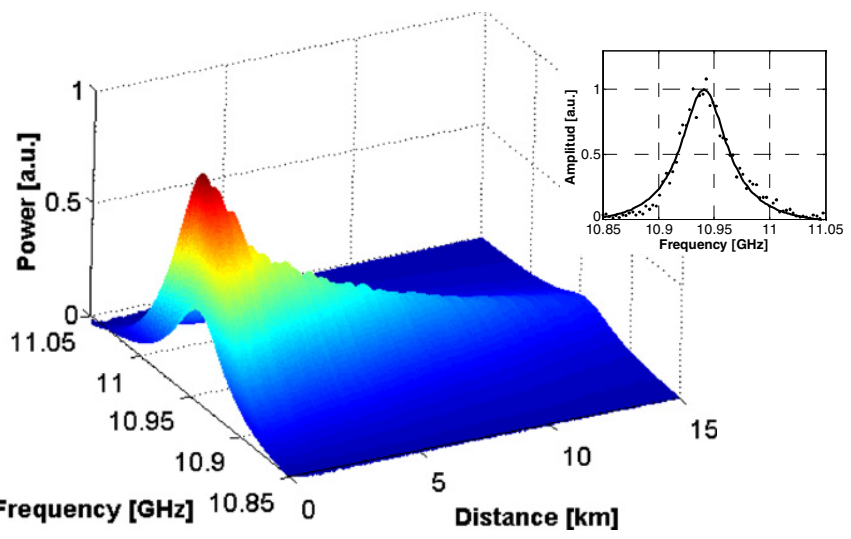

Figure 5. Brillouin signal in electrical domain $(\sim 10.9 \mathrm{GHz})$. Inset: Lorentzian fitting (with multi-Lorentzian curve) of the spectrum at $5 \mathrm{~km}$.

signal is produced at around $10.94 \mathrm{GHz}$, as shown in figure 5. This signal lies within the Brillouin gain bandwidth, and represents the summed contribution of Stokes and antiStokes components generated by all FP modes, allowing for the simultaneous detection of the average SpBS spectrum (and then the average BFS) with a single photo-detector, and leading then to an improved SNR with respect to the detection of a single longitudinal mode.

Since the result of the proposed multi-mode heterodyne detection scheme for SpBS light is the sum of Brillouinscattering contributions from several FP longitudinal modes (each contribution having the typical Lorentzian-curve spectrum of SpBS), a correct Lorentzian fitting based on the detected spectrum is necessary to take into account the slight wavelength dependence of the SpBS peak for each FP mode [10], also showing some impact on measurement accuracy. For this reason, assuming that the Brillouin gain bandwidth is not significantly altered by different FP modes, the photo-detected Brillouin spectrum when using a FP laser can be expressed as follows:

$$
S(f)=\sum_{i} A_{i} \cdot \frac{W}{W^{2}+\left(f-f_{0}-f_{i}\right)^{2}},
$$

where $S(f)$ is the measured electrical spectrum, $f_{0}$ is the unknown BFS parameter for the central FP mode wavelength, $W$ is the gain bandwidth, $A_{i}$ is the weight of the $i$ th FP laser mode power, and $f_{i}$ is the slight shift of Brillouin peak for each mode (estimated from FP mode separation and optical fiber data).

Hence, the measured electrical spectrum is fitted with the multiple Lorentzian curve in (4) at each fiber position, so that the central BFS is obtained. An example of the fitted spectrum versus fiber distance for FP laser is reported in figure 5, also showing an example of the envelope data fitting taken at $5 \mathrm{~km}$ fiber distance (see figure 5 inset), where it can be noted that data fitting results in a Brillouin FWHM which is larger than typical Brillouin linewidth.

\section{Results}

In order to measure the Brillouin spectrum profile as a function of the distance along the sensing fiber, the coherent 


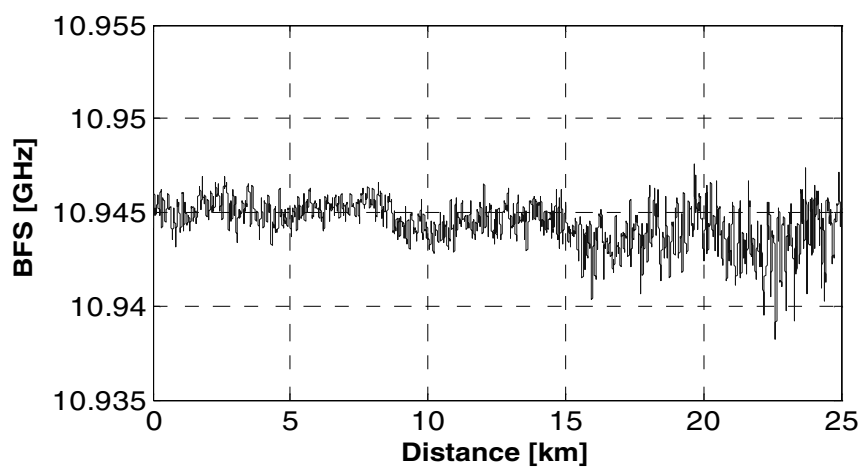

Figure 6. BFS measured along the sensing fiber.

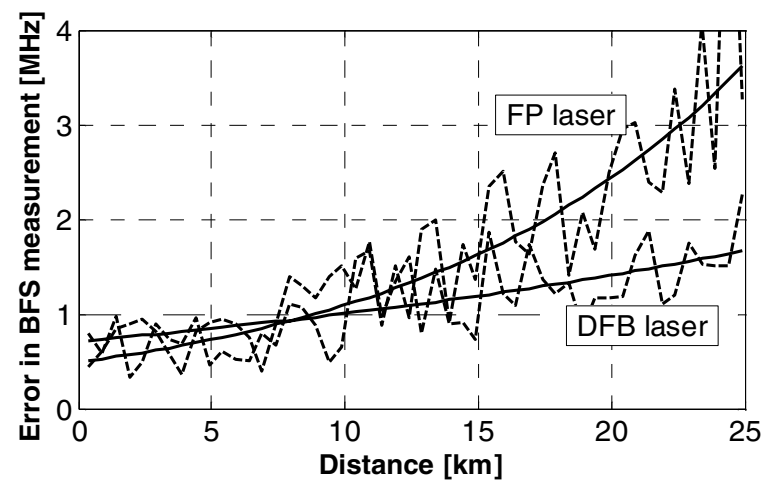

Figure 7. Error in BFS measurement versus distance, for DFB and FP lasers.

self-heterodyne detector of figure 2 has been used together with a fast acquisition system for each wavelength interval. The minimum attainable spatial resolution of $\sim 35 \mathrm{~m}$ is dictated by the largest available resolution bandwidth of the ESA operating in zero-span mode $(3 \mathrm{MHz})$. The Brillouin spectral shape along the sensing fiber is then obtained by frequency stepping within the Brillouin gain bandwidth, and by fast acquisition of the corresponding $z$-dependent trace.

While the used DFB laser is a single-longitudinal mode laser with a good single-mode suppression ratio $(>40 \mathrm{~dB})$, the FP laser employed in the experiment has about 30 longitudinal modes ( $\sim 7 \mathrm{dBm}$ power per mode), each one able to generate a couple of Stokes and anti-Stokes SpBS components. The same laser is also used as a multi-wavelength OLO, and hence it is mixed at the PIN photodiode together with the SpBS components that are backscattered from the sensing fiber (both signals are shown in figure 4, with normalized peak power) without the need for optical filtering of Rayleigh-scattering in the SpBS light branch. An example of the BFS trace versus fiber distance, obtained as detailed above, is shown in figure 6, for an unstrained optical fiber placed at room temperature. The graph in figure 7 describes the attained experimental rms error in the measured BFS versus fiber distance, for both FP and DFB lasers. From figure 7 it can be seen that although the power of the used FP laser is notably higher than that of DFB (theoretically allowing for better accuracy in BFS measurements), the noise in BFS trace is smaller when using a DFB laser. This result indicates the

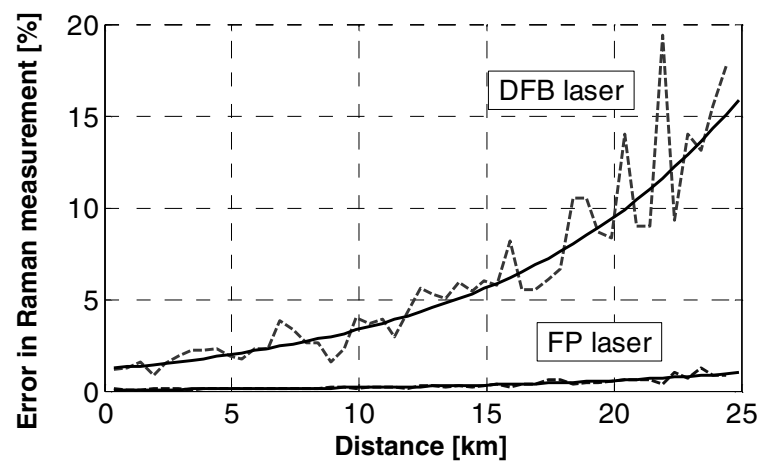

Figure 8. Error in anti-Stokes trace versus distance, for DFB and FP lasers.

occurrence, when employing FP lasers, of additional noise sources that can be related to different optical phenomena affecting the resolution. The two major effects impacting the BFS accuracy when using FP lasers are given by the $\lambda$ dependence in BFS and the frequency drift of the FP modes.

Regarding the first effect, the slight wavelength dependence in BFS (combined with our heterodyne scheme simultaneously detecting the SpBS from many longitudinal modes) generates a detected trace which is the sum of many shifted Lorentzian curves, as detailed in the previous section. The result is that the detected spectrum has a linewidth $\Delta S$ which is larger than the inherent linewidth of the SpBS. The rms noise $\left(\delta v_{B}\right)$ in the BFS depends ultimately on the electrical SNR in the SpBS spectrum and on the spectrum linewidth itself $(\Delta S)$, according to [11]

$$
\delta v_{B}=\frac{\Delta S}{\sqrt{2}(\mathrm{SNR})^{1 / 4}},
$$

implying that an enlargement in the detected linewidth $\Delta S$, with the same SNR, leads to a proportional increase in rms noise. Regarding the second effect, this is linked to the wavelength drift of FP modes within one trace acquisition time $(250 \mu \mathrm{s})$. Actually, since the same laser is used for generating the SpBS as well as for the OLO, it is evident that a wavelength drift would cause impairments in the heterodyne detection. However, the wavelength stability of the optical source is important within a time comparable with the single trace acquisition duration.

Although both sources (FP and DFB) have shown a wavelength stability which is much smaller than the Brillouin gain bandwidth over the $250 \mu$ s timescale, some degree of impairment is however expected in the self-heterodyne detection when using FP lasers (with $\lambda$ drift $<1 \mathrm{MHz}$ over $100 \mu$ s scale) compared to DFB lasers, which typically exhibit a far better wavelength stability $(<$ few hundred $\mathrm{kHz}$ over ms scale).

The graph in figure 8 describes the attained rms noise in the measured anti-Stokes Raman trace versus fiber distance for both FP and DFB lasers. It is evident, in this case, that the high-power and broad bandwidth features of the FP laser enable a notably higher accuracy than when using DFB lasers, with negligible additional impairments. 


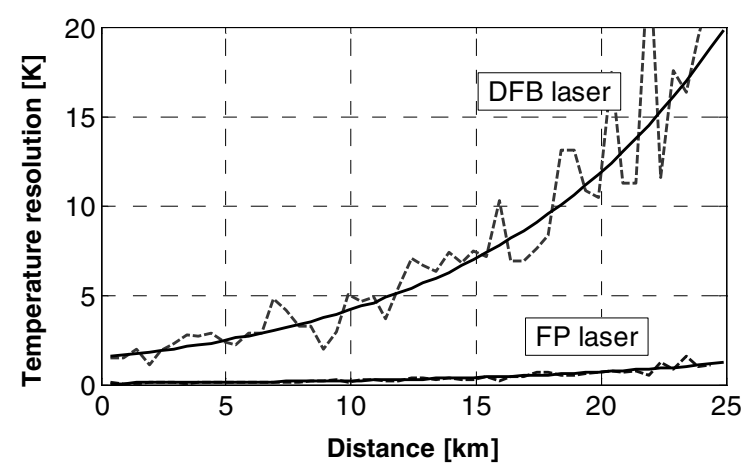

(a)

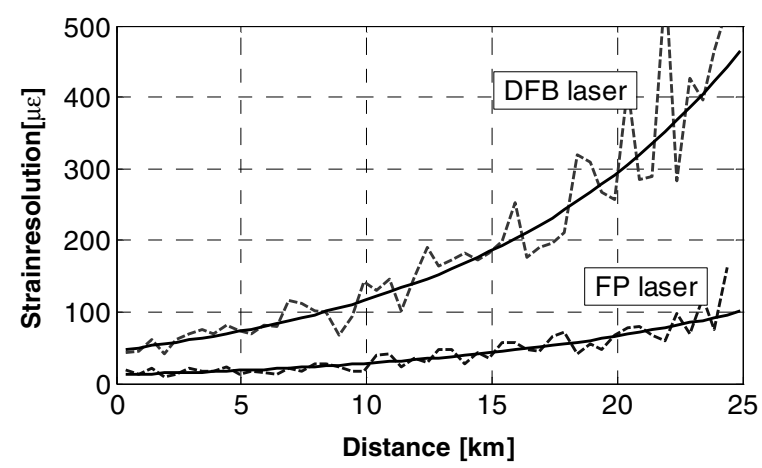

(b)

Figure 9. (a) Temperature resolution and (b) strain resolution versus distance, for both DFB and FP lasers.

Finally, if the overall accuracy improvement is analyzed employing equation (2) to estimate the distributed fiber temperature, and equation (3) to estimate the temperatureindependent fiber strain, notable benefits when using FP lasers can be observed. This is clearly visible in figure 9, showing the temperature and strain resolution along the fiber, where it can be observed that the increased noise in the BFS mentioned before has only a small impact on the ultimate strain and temperature resolutions, which are notably better when employing a FP laser with respect to a DFB laser. This can be simply inferred considering that the limiting factor in hybrid sensing is essentially given [2] by the noise in antiStokes Raman intensity measurement, where the use of the FP laser has a strong beneficial impact (as in figure 9).

A high accuracy in temperature measurements has actually only been achieved when using the FP laser. The achieved temperature resolution $\delta T$ is shown in figure $9(a)$, indicating that a value of $\delta T \sim 20 \mathrm{~K}$ (at $25 \mathrm{~km}$ distance) obtained with a DFB laser can be improved down to $\delta T \sim$ $1.2 \mathrm{~K}$ by using the FP laser (attaining $\delta T<0.2 \mathrm{~K}$ within the first $10 \mathrm{~km}$ of fiber), resulting in about $12 \mathrm{~dB}$ net improvement. Note that in sensor performance analysis, it is not required to apply any actual strain or temperature change to the fiber, since the attainable resolution parameters depend ultimately on the SNR of the measured traces. When calculating the final temperature-independent strain distribution (and hence the strain resolution), the noise in the temperature trace has an overwhelming importance compared to the noise in the BFS trace, and therefore an accuracy enhancement in temperature measurement has a nearly equivalent impact also on the final strain accuracy. Figure $9(b)$ actually indicates that a strain resolution of $\sim 470 \mu \varepsilon$ at $25 \mathrm{~km}$ distance, obtained with the DFB laser, can be improved down to $\sim 100 \mu \varepsilon$ when using the FP laser, resulting in about $7 \mathrm{~dB}$ net improvement.

\section{Conclusion}

In conclusion, the use of multi-wavelength FP lasers has been proposed for achieving simultaneous strain and temperature measurements based on hybrid Raman-Brillouin distributed optical sensors, allowing for significant sensing performance improvement and helping to reduce sensor complexity and cost. The multiple longitudinal modes of FP lasers provide a power distribution along the spectrum, overcoming the limitation of the maximum peak pulse power at the fiber input, imposed by nonlinearities, such as SBS threshold, allowing for further improvement when using optical pulse techniques or optical amplification. Moreover, results show a significant reduction of issues related to coherent Rayleigh noise, thanks to the large FWHM in FP lasers.

It is experimentally shown that the use of FP lasers allows for a significant performance enhancement in hybrid RamanBrillouin sensing, resulting in about $12 \mathrm{~dB}$ improvement in temperature accuracy (and $7 \mathrm{~dB}$ in strain accuracy) at $25 \mathrm{~km}$ compared to standard DFB lasers, and enabling a strain (temperature) resolution of $\sim 100 \mu \varepsilon\left(1.2^{\circ} \mathrm{C}\right)$ at $25 \mathrm{~km}$ distance, compared to $\sim 470 \mu \varepsilon\left(20^{\circ} \mathrm{C}\right)$ obtained with a DFBlaser scheme.

\section{References}

[1] Parker T R, Farhadiroushan M, Handerek V and Rogers A J 1997 A fully distributed simultaneous strain and temperature sensor using spontaneous Brillouin backscatter IEEE Photonics Technol. Lett. 9 979-81

[2] Alahbabi M N, Cho Y T and Newson T P 2005 Simultaneous temperature and strain measurement with combined spontaneous Raman and Brillouin scattering Opt. Lett. 30 1276-8

[3] Bao X, Webb D J and Jackson D A $199332 \mathrm{~km}$ distributed sensor based on Brillouin loss in an optical fiber Opt. Lett. 18 1561-3

[4] Wait P C et al 1997 A theoretical comparison of spontaneous Raman and Brillouin based fibre optic distributed temperature sensors Opt. Commun. 144 17-23

[5] Bolognini G et al 2007 Analysis of distributed temperature sensing based on Raman scattering using OTDR coding and discrete Raman amplification Meas. Sci. Technol. 18 3211-8

[6] De Souza K 2006 Significance of coherent Rayleigh noise in fibre-optic distributed temperature sensing based on spontaneous Brillouin scattering Meas. Sci. Technol. 17 1065-9

[7] Soto M A, Bolognini G and Di Pasquale F 2008 Analysis of optical pulse coding in spontaneous Brillouin-based distributed temperature sensors Opt. Express 16 19097-111

[8] Santoro M A and Poole C D 1994 Polarization scrambling using a short piece of high-birefringence optical fiber and a multifrequency laser diode J. Lightwave Technol. 12 288-93 
[9] Diaz S, Mafang S F, Lopez-Amo M and Thévenaz L 2008 A high-performance optical time-domain Brillouin distributed fiber sensor IEEE Sensors $J$. 8 1268-72

[10] Azuma Y, Shibata N, Horiguchi T and Tateda M 1988 Wavelength-dependence of Brillouin-gain spectra for single-mode optical fibres Electron. Lett. 24 250-2

[11] Horiguchi T, Shimizu K, Kurashima T, Tateda M and Koyamada Y 1995 Development of a distributed sensing technique using Brillouin scattering J. Lightwave Technol. 13 1296-301 